\title{
Serous Retinal Detachment
}

National Cancer Institute

\section{Source}

National Cancer Institute. Serous Retinal Detachment. NCI Thesaurus. Code C118756.

Retinal detachment secondary to fluid accumulation under the neurosensory retina without a retinal tear or break. 